\title{
Rotating Disc around a Schwarzschild Black Hole
}

\author{
Oldřich Semerák *(D) and Pavel Čížek \\ Institute of Theoretical Physics, Faculty of Mathematics and Physics, Charles University, CZ-180 00 Prague, \\ Czech Republic; ciz@matfyz.cz \\ * Correspondence: oldrich.semerak@mff.cuni.cz
}

Received: 14 December 2019; Accepted: 26 January 2020; Published: 3 February 2020

Simple Summary: Metric for a linear perturbation of Schwarzschild due to a rotating thin disc.

\begin{abstract}
A stationary and axisymmetric (in fact circular) metric is reviewed which describes the first-order perturbation of a Schwarzschild black-hole space-time due to a rotating finite thin disc encircling the hole symmetrically. The key Green functions of the problem (corresponding to an infinitesimally thin ring) - the one for the gravitational potential and the one for the dragging angular velocity - were already derived, in terms of infinite series, by Will in 1974, but we have now put them into closed forms using elliptic integrals. Such forms are more practical for numerical evaluation and for integration in problems involving extended sources. This last point mostly remains difficult, but we illustrate that it may be workable by using the simple case of a finite thin disc with constant Newtonian surface density.
\end{abstract}

Keywords: gravitation; relativity; black-hole physics; dragging

\section{Static and Stationary Sources around Black Holes}

Many highly active sources in the Universe appear to be driven by black holes strongly interacting with the surrounding matter and fields. Black holes are well accessible to analytical study if they are isolated and stationary (under asymptotic flatness, such assumptions lead to the Kerr-Newman family of solutions), but otherwise it is a formidable task. There exist several special non-stationary black-hole solutions, such as those belonging to the Robinson-Trautman class, but, in general, heavy numerical codes or/and sophisticated approximation techniques have to be employed then. However, stationarity can often be assumed, since-as concerns the gravitational field-most astrophysical systems dominated by black holes remain almost stationary over considerable time periods. On the other hand, it may be problematic to assume isolation, namely to approximate the gravitational field by that solely generated by the black hole, because even a very low-mass (and moderate-density) additional source can be crucial for certain features of the field. For example, in a Schwarzschild space-time, actually any additional source dominates the tangential component of the field, simply because the original field is exactly radial. This is a rather trivial example, but such a conclusion holds quite generally for higher derivatives of the field, at least in the vicinity of the external sources; ${ }^{1}$ actually, it may already hold on the level of curvature (second derivatives of metric). The curvature, conversely, determines the stability of motion of the matter (of the matter itself which contributes to the curvature). Consequently, a (self-)gravitating matter may assume-under otherwise the "same" conditions-quite a different configuration than a test matter.

1 Exactly this is the first-plan message of Einstein's equations: "curvature $\sim$ density". 
In a quasi-stationary situation, the matter flow is supposed to typically have, around astrophysical black holes, a roughly axially symmetric disc geometry. If the matter just follows circular orbits (it moves in the direction of the two space-time symmetries), the space-time is also orthogonally transitive, which means that there exist global meridional planes, everywhere orthogonal to both the Killing symmetries. The metric of such circular space-times (see e.g., [1] for a thorough account) can be written

$$
\mathrm{d} s^{2}=-e^{2 v} \mathrm{~d} t^{2}+B^{2} r^{2} e^{-2 v} \sin ^{2} \theta(\mathrm{d} \phi-\omega \mathrm{d} t)^{2}+e^{2 \zeta-2 v}\left(\mathrm{~d} r^{2}+r^{2} \mathrm{~d} \theta^{2}\right),
$$

where the $t$ and $\phi$ coordinates (time and azimuth) are adapted to the Killing symmetries, while $r$ (called isotropic radius) and $\theta$ cover the meridional surfaces orthogonal to both Killing directions. They are related to the Weyl-type cylindrical coordinates by

$$
\rho=r \sin \theta, \quad z=r \cos \theta \text {. }
$$

The unknown metric functions $\nu, B, \omega$ and $\zeta$ only depend on $r$ and $\theta$; they are determined by the Einstein equations. The equation for $B$ reads, in the Weyl coordinates,

$$
B_{, \rho \rho}+\frac{2 B_{, \rho}}{\rho}+B_{, z z}=8 \pi B\left(T_{\rho \rho}+T_{z z}\right)
$$

and it is being solved first in any specific situation. In a vacuum case $\left(T_{\mu \nu}=0\right)$, and when a black hole (of mass $M$ ) is present, it is convenient to choose

$$
B=1-\frac{M^{2}}{4\left(\rho^{2}+z^{2}\right)}=1-\frac{M^{2}}{4 r^{2}} ;
$$

with such a choice, the horizon lies at $r=M / 2$ (while the other common choice $B=1$ makes the horizon just a rod at $\rho=0,|z| \leq M$, which is less convenient).

Provided that the energy-momentum tensor satisfies $T_{\rho}^{\rho}+T_{z}^{z}=0$, the remaining independent field equations can be combined to

$$
\begin{gathered}
\nabla \cdot(B \nabla v)-\frac{B^{3} \rho^{2}}{2 e^{4 v}}(\nabla \omega)^{2}=4 \pi B e^{2 \zeta-2 v}\left(T_{\phi}^{\phi}-2 \omega T_{\phi}^{t}-T_{t}^{t}\right), \\
\nabla \cdot\left(B^{3} \rho^{2} e^{-4 v} \nabla \omega\right)=-16 \pi B e^{2 \zeta-2 v} T_{\phi}^{t}, \\
\zeta_{, \rho}(B \rho)_{, \rho}-\zeta_{, z}(B \rho)_{, z}=-B \rho\left[\left(v_{, \rho}\right)^{2}-\left(v_{, z}\right)^{2}\right]-\frac{1}{2}\left[(B \rho)_{, \rho \rho}-(B \rho)_{, z z}\right]+\frac{1}{4} B^{3} \rho^{3} e^{-4 v}\left[\left(\omega_{, \rho}\right)^{2}-\left(\omega_{, z}\right)^{2}\right], \\
\zeta_{, \rho}(B \rho)_{, z}+\zeta_{, z}(B \rho)_{, \rho}=-2 B \rho v_{, \rho} v_{, z}-(B \rho)_{, \rho z}+\frac{1}{2} B^{3} \rho^{3} e^{-4 v} \omega_{, \rho} \omega, z,
\end{gathered}
$$

where $\nabla$ and $\nabla$. denote gradient and divergence in an (auxiliary) Euclidean three-space. Appropriate boundary conditions at infinity (asymptotical flatness), at the horizon (regularity) and on the axis (absence of conical singularity) have to be added. The first two equations should be solved for $v$ and $\omega$, and then-with $v$ and $\omega$ already known $-\zeta$ can be obtained by line integration from the last two equations.

Practically, the above procedure is almost never feasible analytically, because the equations are non-linear and coupled. The only simple exception is the static case, $\omega=0$, when, outside of the sources, (4) becomes the Laplace equation. Therefore, in the static case, $v$ behaves exactly like the Newtonian potential; in particular, $v$ generated by multiple sources is obtained by linear superposition. The exercise still differs from the Newtonian one, because there is also the second metric function $\zeta$. Equations (6) and (7) simplify considerably in the static case, yet they still remain non-linear (in gradient of $v$ ) and their line integration can usually be only done numerically. However, $\zeta$ influences the meridional geometry, and it can actually deform it very strongly with respect to the corresponding 
(flat) Newtonian situation (described by the same $v$ ). As expected, the biggest differences arise in the vicinity of very compact sources where the gradient of $v$ is very large.

In the generic, stationary but non-static case, there are still two main analytical options. The first one is to use some of the generating techniques - mathematical operations which transform between metrics of the given symmetries. It is known that actually any solution can be obtained in this way, but one has little control over what comes out-most of such solutions remain unexplored, and most of them are likely to be unphysical. The second option is to perform, within the given class of metrics, a perturbation of some known solution. This route is much more straightforward, but it is only limited to small deviations from the chosen "seed" metric. ${ }^{2}$ Also, even for very simple seeds, the equations are still so complicated that it is usually impossible to reach more than a linear order.

\section{Subject of This Paper}

In the present paper, we report some recent results on perturbative treatment of circular (stationary, axisymmetric and orthogonally transitive) problem, starting from the Schwarzschild space-time as the seed. The Green functions for $v$ and $\omega$ were already given by Will in 1974 [2] (they correspond to the linear perturbation due to a slowly rotating and light circular ring of infinitesimal cross section). The Green functions, written there in terms of infinite series, we put into closed forms using elliptic integrals. ${ }^{3}$ Such forms are better for numerical study, but mainly they are more convenient when trying to solve problems involving extended sources (when the Green functions have to be integrated out, together with the respective density, over the source volume). We demonstrated that such an integration can in simple cases really be performed, on the example of a linear perturbation of the Schwarzschild black hole due to a constant-density finite thin circular disc extending between two concentric radii. We also showed that the solution has satisfactory properties within a reasonable parameter range, in particular, that the disc can be interpreted either as a one stream of ideal fluid or as two counter-rotating dust (geodesic) streams.

Below, we first (Section 2) write down the formulas describing the above linear perturbation, then in Section 3 we list basic properties of the solution (showing that it is physically acceptable), and finally add some comments and future options in Concluding remarks. For details of the derivation, as well as for literature, we refer to the papers [5,6].

Notation: we use geometrized units in which $c=1, G=1$, index-posed comma indicates partial differentiation and usual summation rule is employed. Space-time metric $g_{\mu \nu}$ has signature $(-+++)$.

\section{Metric for the Rotating-Disc Perturbation of Schwarzschild}

Let us stress once more that the perturbation we consider is special in keeping the space-time circular (i.e., stationary, axisymmetric and possessing global meridional planes). The procedure is quite straightforward: we took the metric (1), chose $B$ according to (3), expressed the other metric functions $v, \omega$ and $\zeta$ as power series in a small parameter proportional to the mass density of the perturbing source, expanded the source terms themselves in a similar way, wrote down the Einstein equations, linearized them in the perturbation quantities, found the Green functions for the perturbations of $v$ and $\omega$, and expressed the latter in close forms. Such forms are a natural starting point when trying to find the field of extended sources ${ }^{4}$ (by convoluting the Green functions with the source terms). Not to

2 The deviation in fact need not be small: instead of "perturbation", one can speak of a solution of the equations in terms of series. However, even in such a more general case the series have to converge reasonably.

3 The Green function of the potential $v$ had actually been known long before; it describes what in general relativity is being called the Bach-Weyl ring [3]. That solution is a good example of how the second metric function $\lambda$ (known analytically in this case) can deform the original "Newtonian" solution in a weird way [4].

4 By extended, we mean spatially more than one-dimensional. Actually, in general relativity, the dividing line between "physically intelligible" and other sources generally lies between lines and shells—see [7]. 
forget, if successful in finding the perturbed $v$ and $\omega$, one should also finally fix $\zeta$ by a line integral given by the gradients of (the already known) $v$ and $\omega$. See [5] for details.

The specific result we summarize here for illustration is the linear perturbation of Schwarzschild due to a rotating thin disc lying between two radii $r_{\text {out }}>r_{\text {in }}(>M / 2)$. For the gravitational potential $v$, we employ the known formula for the potential generated by a uniform-density disc in the Newtonian theory, see [8]. It leads to the expression ${ }^{5}$

$$
v(x, \theta)=v_{\mathrm{Schw}}+V\left(x^{\prime}=x_{\mathrm{out}} ; x, \theta\right)-V\left(x^{\prime}=x_{\mathrm{in}} ; x, \theta\right),
$$

where

$$
v_{\mathrm{Schw}}=\ln \frac{2 r-M}{2 r+M}
$$

is the Schwarzschild part, and the potential of the disc (of constant Newtonian surface density $S$ ) reads

$$
\begin{aligned}
V\left(x^{\prime} ; x, \theta\right)= & 2 \pi M S x|\cos \theta| H\left(x^{\prime 2}-\cos ^{2} \theta-x^{2} \sin ^{2} \theta\right)-2 M S \sqrt{a_{31} a_{42}} E(k)- \\
& -\frac{2 M S}{\sqrt{a_{31} a_{42}}}\left(x^{\prime 2}-\cos ^{2} \theta-x^{2} \sin ^{2} \theta\right) \times \\
& \times\left[K(k)+\frac{x^{2} \cos ^{2} \theta}{\left(\sqrt{x^{\prime 2}-1}+\sqrt{x^{2}-1} \sin \theta\right)^{2}} \Pi\left(\frac{4 \sqrt{\left(x^{2}-1\right)\left(x^{\prime 2}-1\right)} \sin \theta}{\left(\sqrt{x^{\prime 2}-1}+\sqrt{x^{2}-1} \sin \theta\right)^{2}}, k\right)\right],
\end{aligned}
$$

where $x:=\frac{r}{M}\left(1+\frac{M^{2}}{4 r^{2}}\right)$ is a suitable dimensionless radius, $H(x)$ stands for the Heaviside function, the $a_{r s}$ and $k$ are given by

$$
\begin{aligned}
& a_{1}=x x^{\prime}+\sqrt{\left(x^{2}-1\right)\left(x^{\prime 2}-1\right)}, \quad a_{2}=x x^{\prime}-\sqrt{\left(x^{2}-1\right)\left(x^{\prime 2}-1\right)}, \quad a_{3}=\sin \theta, \quad a_{4}=-\sin \theta \\
& a_{r s}=a_{s}-a_{r}, \quad k^{2}=\frac{2 a_{21} \sin \theta}{a_{31} a_{42}}=\frac{4 \sqrt{\left(x^{2}-1\right)\left(x^{\prime 2}-1\right)} \sin \theta}{x^{2} x^{\prime 2}-\left[\sqrt{\left(x^{2}-1\right)\left(x^{\prime 2}-1\right)}-\sin \theta\right]^{2}}
\end{aligned}
$$

and $K(k), E(k)$ and $\Pi(., k)$ are standard complete elliptic integrals ( $k$ is their modulus, so the roots in definitions contain $\left.k^{2}\right)$.

The dragging angular velocity $\omega$ is fully given by the linear perturbation (since $\left.\omega_{\text {Schw }}=0\right){ }^{6}$

$$
\begin{aligned}
& (x+1)^{3} \omega(x, \theta)=\frac{W}{2}\left[\frac{1}{\left(x_{\text {in }}+1\right)^{2}}-\frac{1}{\left(x_{\text {out }}+1\right)^{2}}\right]-\frac{x-1}{1+|\cos \theta|}\left[\frac{x_{\text {in }}}{\left(x_{\text {in }}+1\right)^{2}}-\frac{x_{\text {out }}}{\left(x_{\text {out }}+1\right)^{2}}\right]+ \\
& +\frac{W}{2 \pi \sin ^{2} \theta}\left[a_{41} \frac{Y_{\mathrm{K}} K(k)+\gamma_{\mathrm{E}} E(k)+Y_{0} \Pi\left(n_{0}, k\right)+Y_{1} \Pi\left(n_{1}, k\right)+Y_{2} \Pi\left(n_{2}, k\right)}{\left(x^{\prime}+1\right)^{2} \sqrt{a_{31} a_{42}}}\right]_{x_{\text {in }}}^{x_{\text {out }}},
\end{aligned}
$$

where $W$ is a free constant (like $S$ in the case of potential $v$ ), the factors

$$
n_{0}=-\frac{2 \sin \theta}{a_{31}}, \quad n_{1}=-\frac{2 \sin \theta\left(a_{1}+1\right)}{a_{31}(1-\sin \theta)}, \quad n_{2}=\frac{2 \sin \theta\left(a_{1}-1\right)}{a_{31}(1+\sin \theta)}
$$

5 We use the same notation as in the original paper [5], only omitting the subscript " 1 " which we used there to indicate the first perturbation order.

6 The expression is simplified considerably with respect to the original paper [5]. However, please note that a mistake occurred in that paper in the expression for $I_{3}\left(x^{\prime}\right)$ in equation (86): instead of the factor $\left[x^{2} x^{\prime 2}+\cos ^{2} \theta-\left(x-x^{\prime}\right)^{2}\right]$, there should be $\left[3 x^{2} x^{\prime 2}-x^{2}-x^{\prime 2}+\cos ^{2} \theta\right]$ in the numerator in front of the elliptic integral $\Pi\left(-\frac{2 \sin \theta}{a_{31}}, k\right)$. The mistake had no effect on the rest of the paper, and it also did not occur in numerical-evaluation codes, so the figures therein are correct. 
represent characteristics of the $\Pi(n, k)$ integrals, the "coefficients" standing at elliptic integrals read

$$
\begin{aligned}
& Y_{\mathrm{K}}=\frac{a_{31}}{a_{1}^{2}-1}\left[2 x^{\prime}(x-1)\left(x^{\prime} a_{1}+1\right)+x a_{21}-x x^{\prime}\left(x^{2}-x^{\prime 2}\right)\right] \\
& Y_{\mathrm{E}}=\frac{x^{\prime}(x-2) a_{31} a_{42}}{a_{41}} \\
& Y_{0}=-2(x-1)\left(x^{\prime 2}+1\right)-2 \sin ^{2} \theta \\
& Y_{1}=-\frac{(x-1)\left(x^{\prime}-1\right)^{2}\left(x+x^{\prime}\right)(1+\sin \theta)}{a_{1}+1} \\
& Y_{2}=\frac{(x-1)\left(x^{\prime}+1\right)^{2}\left(x-x^{\prime}\right)(1-\sin \theta)}{a_{1}-1}
\end{aligned}
$$

and the evaluation at the disc edges has been denoted by $[X]_{x_{\text {in }}}^{x_{\text {out }}}=X\left(x^{\prime}=x_{\text {out }}\right)-X\left(x^{\prime}=x_{\text {in }}\right)$.

Finally, the last metric function $\lambda$ can only be found numerically. The easiest way is to write down the relevant Einstein equations in the Weyl coordinates $(\rho, z)$ and with the choice $B=1$. In vacuum and in the linear perturbation order (when the function $\omega$ does not enter at all), they are the same as in the static case,

$$
\lambda_{, \rho}=\rho\left[\left(v_{, \rho}\right)^{2}-\left(v_{, z}\right)^{2}\right], \quad \lambda_{, z}=2 \rho v_{, \rho} v_{, z}
$$

These equations are solved by a line integration going from the axis (where $\lambda=\ln B$ necessarily, so for $B=1$ it vanishes) to a given location along any path going through a vacuum region. However, in order to be consistent with the above results, one then has to adapt the solution to our choice $B=1-\frac{M^{2}}{4 r^{2}}$. This is done according to the transformation (see [5], Equation (80); note that the first expression for $\tilde{\rho}$ was given there with a wrong sign)

$$
\begin{aligned}
\tilde{\rho} & =\frac{\rho\left(4 \rho^{2}+4 z^{2}-M^{2}\right)}{4\left(\rho^{2}+z^{2}\right)}=\frac{\left(4 r^{2}-M^{2}\right) \sin \theta}{4 r}=M \sqrt{x^{2}-1} \sin \theta, \\
\tilde{z} & =\frac{z\left(4 \rho^{2}+4 z^{2}+M^{2}\right)}{4\left(\rho^{2}+z^{2}\right)}=\frac{\left(4 r^{2}+M^{2}\right) \cos \theta}{4 r}=M x \cos \theta, \\
\tilde{\lambda} & =\lambda-\frac{1}{2} \ln \frac{\left(4 \rho^{2}+4 z^{2}+M^{2}\right)^{2}-16 M^{2} z^{2}}{16\left(\rho^{2}+z^{2}\right)^{2}}=\lambda-\frac{1}{2} \ln \frac{\left(4 r^{2}+M^{2}\right)^{2}-16 r^{2} M^{2} \cos ^{2} \theta}{16 r^{4}}= \\
& =\lambda-\frac{1}{2} \ln \frac{4\left(x^{2}-\cos ^{2} \theta\right)}{\left(x+\sqrt{x^{2}-1}\right)^{2}},
\end{aligned}
$$

where tilded quantities correspond to $B=1$ and untilded quantities to $B=1-\frac{M^{2}}{4 r^{2}}$.

\section{Basic Properties of the Solution}

\subsection{Mass and Angular Momentum}

Let us list basic properties of the above linear-perturbation solution. The mass and angular momentum can either be found by computing the respective Komar integrals, or from behaviour of the metric functions at radial infinity. Both methods yield the same results, in particular, the metric functions fall off asymptotically $(r \rightarrow \infty)$ as

$$
v \propto-\frac{M+\mathcal{M}}{r}, \quad \omega \propto \frac{2 \mathcal{J}}{r^{3}}, \quad \zeta \propto-\frac{M^{2}}{4 r^{2}}
$$


(the last relation can easily be obtained on the symmetry axis where $e^{\zeta}=B$ must hold), where

$$
\mathcal{M}=\pi S\left[r_{\text {out }}^{2}-r_{\text {in }}^{2}-\frac{M^{4}}{16}\left(\frac{1}{r_{\text {in }}^{2}}-\frac{1}{r_{\text {out }}^{2}}\right)\right], \quad \mathcal{J}=\frac{W M^{2}}{8}\left[r_{\text {out }}-r_{\text {in }}-\frac{M^{2}}{4}\left(\frac{1}{r_{\text {in }}}-\frac{1}{r_{\text {out }}}\right)\right]
$$

represent the disc contributions, while the black-hole mass remains $M$ and the black-hole angular momentum remains zero. In spite of the latter, the horizon does rotate with respect to infinity with non-zero angular velocity,

$$
\omega_{\mathrm{H}}=\frac{W}{8} \frac{\left(x_{\mathrm{out}}-x_{\mathrm{in}}\right)\left(x_{\mathrm{out}}+x_{\mathrm{in}}+2\right)}{\left(x_{\mathrm{out}}+1\right)^{2}\left(x_{\mathrm{in}}+1\right)^{2}} ;
$$

this means that the black hole is just being dragged along by the rotational effect of the disc.

\subsection{Horizon Geometry}

Whenever a black hole is under the (gravitational) influence of some other source, it is interesting to check how affected is the geometry of the horizon (understood as a $2 \mathrm{D}$ surface, namely its $t=\mathrm{const}$ section). Actually, with the choice $B=1-\frac{k^{2}}{4 r^{2}}$, the black-hole horizon stays at $r=\frac{M}{2}$, so its coordinate picture is exactly spherical irrespectively of the perturbation, yet the intrinsic shape of the horizon (given by proper distances in the two angular directions) does change due to the presence of the additional source. In our case, we showed that-in agreement with common experience-the horizon inflates towards the encircling disc, i.e., it becomes oblate. If the disc density $(S)$ is sufficiently large, and/or if the disc is sufficiently extended, the horizon can even be deformed so strongly that its Gauss curvature becomes negative (first at the axis). ${ }^{7}$

The horizon area and surface gravity come out

$$
A_{\mathrm{H}}=16 \pi M^{2} e^{-2 v_{1}(x=1, \theta=0)}=16 \pi M^{2} e^{4 \pi M S\left(x_{\text {out }}-x_{\text {in }}\right)}, \quad \kappa_{\mathrm{H}}=\frac{4 \pi M}{A_{\mathrm{H}}} .
$$

Note that this result is independent of $\theta$, that is, $\kappa_{\mathrm{H}}$ is uniform all over the horizon, in agreement with the zeroth law of black-hole thermodynamics. We see that the horizon area grows (exponentially) while the surface gravity weakens with the increase of the disc density $S$.

\subsection{Static Limit and Singularity}

A rotating horizon is usually surrounded by a static limit-a surface which limits the possibility to stay at rest relative to an asymptotic rest frame (namely to "resist" rotational dragging caused by the source). It is given by $g_{t t}=0$. In a non-rotating case, the static limit coincides with the horizon. We showed that our first-order perturbation does not separate a static limit from the horizon, so there does not appear any ergosphere. Similarly, we also checked, by analysing the Kretschmann scalar (square of the Riemann tensor), that the physical singularity inside the black hole keeps its original, point-like character (and does not turn ring-like as is the case for the Kerr black hole).

\subsection{Circular Motion in the Black-Hole + Disc Field}

The disc influence naturally reveals the properties of test motion. A default first check is the stationary motion along circular orbits, i.e., the motion which just follows the given space-time symmetries. One can for example ask about light-like limits of circular motion, about conditions for

7 This only happens when the disc is very dense/massive, which does not seem to be within the validity of linear perturbation. However, exactly in the linear approximation, the horizon deformation is fully represented by the potential $v$, which-in the linear approximation-superposes just like in the static (non-rotating) case; and in that case, the superposition is exact. 
free (geodesic) circular motion, about zero-speed limit of free circular motion, ${ }^{8}$ about the locations of photon (light-like), marginally stable and marginally bound circular geodesics. We will not list all these properties here, since the explicit forms of the corresponding conditions (specific to our system) are rather cumbersome; they can be found in [6], including illustrations. However, the circular motion is interesting in that it "feels" the rotational perturbation already in the linear order-namely, the respective conditions typically contain the dragging angular velocity $\omega$ linearly.

\subsection{Interpretation of the Disc}

One of the most important questions is whether the resulting general relativistic solution can really be interpreted as describing the field of a black hole encircled by a thin disc with physically acceptable properties. There are two main options how to interpret the disc matter: (i) as a one stream of ideal fluid characterized by proper surface density $\sigma$, proper azimuthal pressure $P$ and orbital velocity $v$ (thus being kept on its circular orbits by a combination of gravitational, inertial and pressure effects); (ii) as two counter-rotating geodesic (i.e., non-interacting, pressureless) streams characterized by their proper densities $\sigma_{ \pm}$and velocities $v_{ \pm}$. As derived in [5], these parameters are given by

$$
\begin{gathered}
\sigma_{ \pm}=\frac{4 r^{2}-8 M r+M^{2}}{8 r^{2}}\left[S \pm \frac{W(M r)^{3 / 2}}{2 \pi(2 r+M)^{3}}\right], \\
v_{ \pm}= \pm v_{0}:= \pm \frac{2 \sqrt{M r}}{2 r-M} ; \\
\sigma=+\frac{\sigma_{+}+\sigma_{-}}{2}+\sqrt{\left(\frac{\sigma_{+}+\sigma_{-}}{2}\right)^{2}+\frac{4 \sigma_{+} \sigma_{-} v_{0}^{2}}{\left(1-v_{0}^{2}\right)^{2}}}= \\
=+\frac{\sigma_{+}+\sigma_{-}}{2}+\sqrt{\left(\frac{\sigma_{+}+\sigma_{-}}{2}\right)^{2}+\frac{16 M r(2 r-M)^{2} \sigma_{+} \sigma_{-}}{\left(4 r^{2}-8 M r+M^{2}\right)^{2}}}, \\
=-\frac{\sigma_{+}+\sigma_{-}}{2}+\sqrt{\left(\frac{\sigma_{+}+\sigma_{-}}{2}\right)^{2}+\frac{4 \sigma_{+} \sigma_{-} v_{0}^{2}}{\left(1-v_{0}^{2}\right)^{2}}}= \\
v^{2}=\frac{\sigma v_{0}^{2}-P}{\sigma-P v_{0}^{2}}=\frac{4 M r \sigma-(2 r-M)^{2} P}{(2 r-M)^{2} \sigma-4 M r P} .
\end{gathered}
$$

Note that in obtaining $v$ from the last expression, the square root has to be taken with $+/-$ sign in case that $\sigma_{+}>\sigma_{-} / \sigma_{+}<\sigma_{-}$. Note also that $v_{ \pm}$are given by their pure-Schwarzschild values (they represent linear velocities with respect to the local zero-angular-momentum observer).

The above can be summarized as follows (see [5] for details again): the metric we derived possesses non-zero jumps across the equatorial plane within the range $\left(x_{\mathrm{in}}, x_{\text {out }}\right)$. Using the Einstein equations, these jumps correspond to a surface energy-momentum tensor which can be written either as due to a one stream of ideal fluid with parameters $\sigma, P$ and $v$, or as due to two freely counter-orbiting streams of dust with parameters $\sigma_{ \pm}$and $v_{ \pm}$(all these components are in steady circular motion). In order that such an interpretation be really plausible, the parameters have to satisfy several physical conditions.

8 This limit is typically not present for motion around an attractive source, but it may be important if something should orbit between a central source (black hole in our case) and another surrounding source (the disc): if the outer source is strong enough, it may attract the orbiting body more than the central source, which means that then "no angular velocity is low enough" - even a body at rest is pulled outwards. 
The first are energy conditions for the energy-momentum tensor. We required the weak, the dominant and the strong energy conditions, and we found that (i) the one-stream parameters have to satisfy $\sigma \geq|P|$, (ii) the two-stream densities $\sigma_{ \pm}$have to be non-negative. Together with the natural requirement $P \geq 0$ (non-negative azimuthal pressure), we thus have

$$
\sigma \geq P \geq 0, \quad \sigma_{ \pm} \geq 0
$$

By analysing the formulae for the above quantities, one finds that $\sigma_{ \pm} \geq 0$ actually implies the first requirements, and that $\sigma_{ \pm}$may only become negative at $r<(1+\sqrt{3} / 2) M \doteq 1.866 M$ (plus $\sigma_{-}$might also become negative if $W$ were too large relative to $S$ ).

The second major physical requirement is that the matter of the disc move with subluminal speed. For both interpretations, this simply implies that the matter (or its respective component) has to only exist above the respective photon circular geodesic, i.e., that the disc must not lie too close to the horizon. Note finally that if the disc solution was considered in astrophysical models, one should, in addition, restrict to such radii where the circular motion is stable.

\subsection{Illustrations}

A number of illustrations have already been given in the original papers. In [5], we plotted the meridional behaviour of the total potential $v$ and of the dragging angular velocity $\omega$, and the radial profiles of the parameters corresponding to the one-stream and two-stream interpretations of the disc. Since the potential $v$ superposes exactly like in the Newtonian case (or like in static axisymmetric case in general relativity), we think it is rather worth to repeat here the other two, "non-trivial" graphs-see Figures 1 and 2.

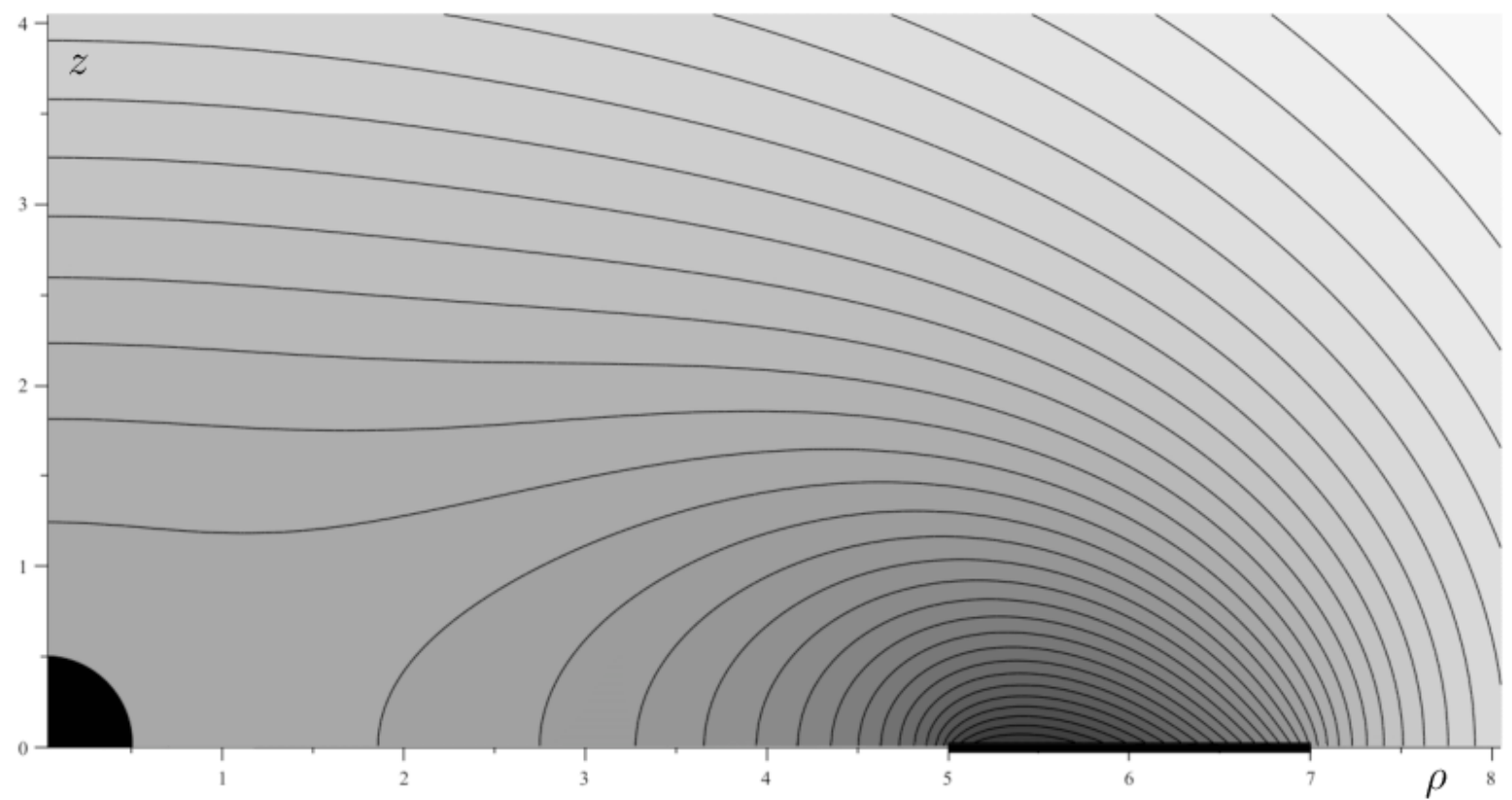

Figure 1. Meridional-plane contours of the dragging angular velocity $\omega$, as entirely given by the first-order perturbation. The disc lies between $\rho=5 M$ and $\rho=7 M$ (it is indicated by thick black line). The angular velocity is everywhere positive, with light/dark shading indicating smaller/larger values (the value reaches about $0.0268 / M$ at the disc and falls off to some $0.0047 / M$ at top right of the plot); the black quarter-disc at $\rho^{2}+z^{2} \leq M^{2} / 4$ is the black hole. Both axes are given in the units of $M$. Since $\omega$ is proportional to $W$, its isolines have the same shape for any $W$. Reprinted from [5] by courtesy of the MNRAS journal. 


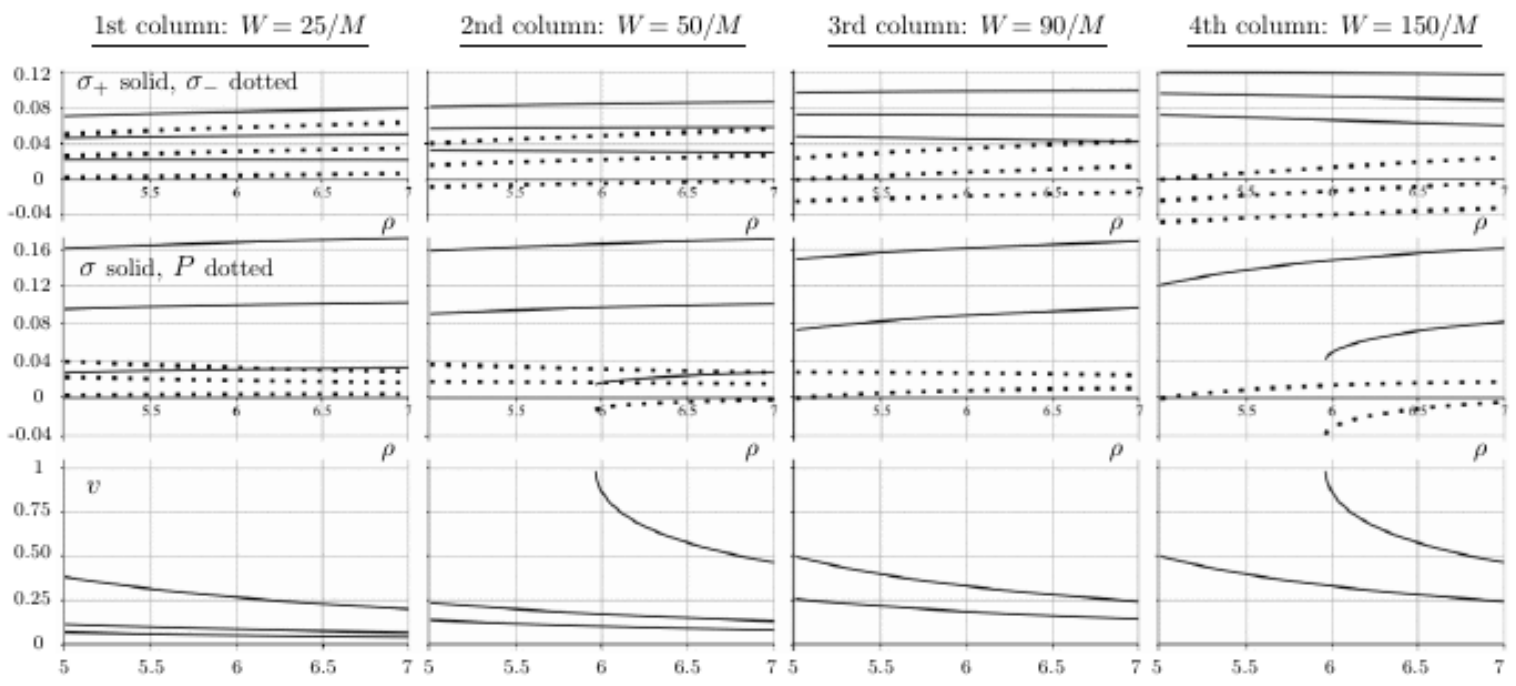

Figure 2. Parameters of the double-stream and single-stream interpretations of the disc, plotted as functions of the Weyl radius $\rho$ for a disc lying between $\rho=5 \mathrm{M}$ and $\rho=7 \mathrm{M}$, for several combinations of the parameters $S$ (Newtonian mass density) and $W$ (scaling the disc rotation). The first / second / third / fourth columns represent the cases given by $W=25 / 50 / 90 / 150$, with the first row showing densities $\sigma_{+}$(solid line), $\sigma_{-}$(dotted line) of the double-stream, counter-rotating dust interpretation, the second row showing density $\sigma$ (solid line) and azimuthal pressure $P$ (dotted line) of the single-stream fluid interpretation, and the third row showing the corresponding "bulk" velocity of the fluid $v$ (solid). For each of the $W$ values, three different values of $S$ have been chosen, $S=0.04,0.12$ and 0.2. The density and pressure curves obtained for higher $S$ are higher (given by larger values), whereas the corresponding bulk velocities decrease with growing $S$. The chosen values of $W$ and $S$ are well out of the scope of the linear perturbation, but this is in order to illustrate clearly what they represent. Since all the parameters (densities, pressure, velocity) should be real and positive, and the velocity $v$ has to be $<1$ in addition, one sees immediately that (i) the discs with a given mass (thus $\rho_{\text {in, }} \rho_{\text {out }}$ and $S$ ) cannot bear however large angular momentum (with growing $W$, the density $\sigma_{-}$of the double-stream interpretation-and consequently also the single-stream parameters-tend to turn negative); and, (ii) since large $W$ implies the need for high orbital velocities, for too large $W$ the orbital interpretation would have to involve superluminal motion (in the bottom row, from left to right, one sees that all three / two / two / one of the shown cases are "physical" in this respect). The densities $S, W, \sigma_{+}, \sigma_{-}$, $\sigma$ as well as the pressure $P$ have the dimension of $1 /$ length and their values are in the units of $1 / M$, the speed $v$ is dimensionless. Reprinted from [5] by courtesy of the MNRAS journal.

Figure 1 shows that the dragging angular velocity decreases when receding from the disc, while staying rather constant in the inner region containing the black hole. It behaves reasonably, including at the very $\operatorname{disc}\left(\theta=\pi / 2, r_{\text {in }}<r<r_{\text {out }}\right)$ where its normal gradient stays finite, namely

$$
\lim _{\theta \rightarrow \frac{\pi}{2}^{-}} \frac{\partial \omega_{1}}{\partial \theta}\left(=-r \lim _{z \rightarrow 0^{+}} \frac{\partial \omega_{1}}{\partial z}\right)=\frac{W}{2} \frac{x-1}{(x+1)^{3}}=8 W M^{2} r^{2} \frac{(2 r-M)^{2}}{(2 r+M)^{6}}
$$

As it is common with thin layers, the only irregularity is in radial gradient at the disc edges.

Figure 2 illustrates radial profiles of the quantities employed in the one-stream and two-stream interpretations of the disc. It is seen that both these interpretations are viable if the parameters $S$ and $W$ are not too large (see the figure caption for details). 


\section{Concluding Remarks}

\subsection{Validity of the Linear Approximation}

Linear-perturbation approximation means that one neglects all terms quadratic and higher-order in the perturbation of $v$ and $\omega$ as well as in any of their derivatives. Validity of such a result is, roughly speaking, restricted to regions where the perturbations of $v$ and $\omega$ as well as their derivatives are small with respect to the unperturbed potential $v_{\mathrm{Schw}}$. Whether this holds depends on where the disc is placed-at large radii where the black-hole influence is already weak, even a very low values of the densities $S$ and/or $W$ can make the disc effect dominant, mainly in its vicinity. However, one is rather motivated by astrophysical accretion discs which are supposed to have their inner radii around or somewhat below $10 \mathrm{M}$.

In [6], we gave a rough observation for a disc lying between $r_{\text {in }}=5 M$ and $r_{\text {out }}=8 M$ (which is just above the pure-Schwarzschild innermost stable circular orbit). In such a configuration, the linear approximation is valid up to some $S \simeq 0.002 / M$ and up to some $W \simeq 20 / M$ : for such values, the disc potential and the dragging function are at worst (close to the disc) about $3 \times$ smaller than $v_{\text {Schw }}$ (in magnitudes of course), so the neglected quadratic terms are at least $10 \times$ smaller. To have an idea, the $5 M<r<8 M$ disc with $S \simeq 0.002 / M$ and $W \simeq 20 / M$ has mass about $\mathcal{M}_{1} \simeq 0.25 M$ and angular momentum about $\mathcal{J}_{1} \simeq 7.45 M^{2}$.

It is important to also check the gradient of $\omega$, because it is the gradient squared through which $\omega$ enters the equation for $v$, see (4). Besides that, one should be cautious if using higher (than the first) derivatives of $\omega$, because dragging generally falls off much faster than potential when receding from the source, so these derivatives do not tend to be small, at least close to the disc (especially close to its edges).

\subsection{Outlook}

It would of course be desirable to proceed to quadratic perturbation order, where only the self-gravitation occurs. In particular, only then $\omega$ "back-reacts" on $v$ (through its gradient squared). Unfortunately, it is almost hopeless to achieve, analytically, the quadratic approximation.

Another possible extension would be to consider a disc with different-than-constant surface density. However, one can hardly hope to be able to integrate out, together with the Green functions, anything else than just very special density profiles.

Even without further generalizations, the above metric for a Schwarzschild black hole encircled by a light rotating thin disc could now be applied (and thus further studied) in various ways. First, we plan to analyse in it the geodesic dynamics, in order to learn how the dynamics is affected by dragging. Namely, the long-term tendencies of the geodesic flow-in particular its possible tendency to chaos-are very sensitive to details of the gravitational field and, at the same time, astrophysically important. We have been studying the geodesic chaos induced by a disc within (exact) static axially symmetric setting (thus without rotation), see, e.g., [9] for a recent state of our series. It will be interesting to see how the observations made there can be perturbed by dragging, the more so that rather an attenuation of chaos (due to dragging) seems to be reported in the literature. In any case, in order to solve any problem with geodesic chaos, one should describe the gravitational field as accurately as possible. In this respect, and regarding that no appropriate exact solution is available, we would trust the above "proper perturbation" more than any approximation based on weak-field expansion of the Kerr metric.

The hole-plus-disc metric might also be applied directly_to model a gravitational field of some cosmic systems. For instance, it has recently been claimed [10] that the rotational curve of our Galaxy (known with considerably better precision now thanks to the Gaia satellite mission) could be fitted, equally well as by Newtonian dynamics in the field of a disc supplemented by the dark-matter halo, within the general relativistic picture based on the Kerr metric. However, regarding the fast fall off with distance of the dragging effects, one would estimate that for stars orbiting at large radii, rotation of 
the central body (the supermassive black hole in the Galaxy case) is less important than dragging generated by the rotating galactic disc itself. The above reviewed metric might be an option in this direction, since it represents the dragging due to the disc reasonably (although the disc we considered has constant Newtonian surface density which is not the case for galaxies).

Otherwise, the reviewed metric might in general be employed as describing a possible space-time background for studying various physics connected with black holes accompanied by accretion discs. It is a more complicated background than the pure Schwarzschild or Kerr one, so one cannot expect to have similar analytical possibilities, and it is only reasonably applicable for low-mass (or slowly rotating) discs, but it should still be worth to use it in studying such effects in which both the mass and rotation of the disc (i.e., the latter's influence on both the gravitational potential and dragging) could be important at the same time. In particular, the knowledge of Green's functions of the gravitational problem should suggest the corresponding electromagnetic ones. Actually, a disc carrying an azimuthal current and thus generating a magnetic field is the case of clear astrophysical interest.

Author Contributions: The results reviewed here were obtained as a part of the PhD thesis of P.Č., which was suggested and supervised by O.S. The key formula for the dragging angular velocity $\omega$ was entirely derived by P.Č. The present paper has been written by O.S., with that formula simplified to the form (12). All authors have read and agreed to the published version of the manuscript.

Funding: O.S. was supported by the grant GACR-17/13525S of the Czech Science Foundation.

Acknowledgments: We thank Petr Kotlařík for discussions.

Conflicts of Interest: The authors declare no conflict of interest.

\section{References}

1. Heusler, M. Black Hole Uniqueness Theorems; Cambridge University Press: New York, NY, USA, 1996.

2. Will, C.M. Perturbation of a Slowly Rotating Black Hole by a Stationary Axisymmetric Ring of Matter. I. Equilibrium Configurations. Astrophys. J. 1974, 191, 521-532. [CrossRef]

3. Bach, R.; Weyl, H. Neue Lösungen der Einsteinschen Gravitationsgleichungen. B. Explizite Aufstellung statischer axialsymmetrischer Felder. Math. Zeit. 1922, 13, 134-145; reprinted in Gen. Relativ. Grav. 2012, 44, 817-832. [CrossRef]

4. Semerák, O. Static axisymmetric rings in general relativity: How diverse they are. Phys. Rev. D 2016, 94, 104021. [CrossRef]

5. Č́žžek, P.; Semerák, O. Perturbation of a Schwarzschild black hole due to a rotating thin disk. Astrophys. J. Suppl. 2017, 232, 14. [CrossRef]

6. Kotlařík, P.; Semerák, O.; Č́žžek, P. Schwarzschild black hole encircled by a rotating thin disc: Properties of perturbative solution. Phys. Rev. D 2018, 97, 084006. [CrossRef]

7. Geroch, R.; Traschen, J. Strings and other distributional sources in general relativity. Phys. Rev. D 1987, 36, 1017. [CrossRef] [PubMed]

8. Lass, H.; Blitzer, L. The gravitational potential due to uniform disks and rings. Celest. Mech. 1983, 30, $225-228$. [CrossRef]

9. Polcar, L.; Suková, P.' Semerák, O. Free Motion around Black Holes with Disks or Rings: Between Integrability and Chaos-V. Astrophys. J. 2019, 877, 16. [CrossRef]

10. Crosta, M.; Giammaria, M.; Lattanzi, M.G.; Poggio, E. Testing Dark Matter and Geometry Sustained Circular Velocities in the Milky Way with Gaia DR2. arXiv:1810.04445. Available online: https://arxiv.org/abs/1810. 04445 (accessed on 2 February 2020).

(C) 2020 by the authors. Licensee MDPI, Basel, Switzerland. This article is an open access article distributed under the terms and conditions of the Creative Commons Attribution (CC BY) license (http:/ / creativecommons.org/licenses/by/4.0/). 